\title{
Stereoretentive Olefin Metathesis Made Easy: In situ Generation of Highly Selective Ruthenium Catalysts from Commercial Start- ing Materials
}

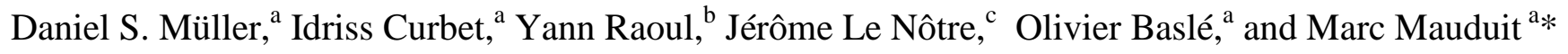 \\ ${ }^{\text {a } U n i v ~ R e n n e s, ~ E c o l e ~ N a t i o n a l e ~ S u p e ́ r i e u r e ~ d e ~ C h i m i e ~ d e ~ R e n n e s, ~ C N R S, ~ I S C R ~ U M R ~ 6226, ~ F-35000 ~ R e n n e s, ~ F r a n c e ~}$ \\ ${ }^{\mathrm{b}}$ OLEON SAS, Venette BP 20609, 60206 Compiègne Cedex, France \\ ${ }^{\text {c } P I V E R T ~ S A S, ~ R u e ~ l e s ~ R i v e s ~ d e ~ l ’ O i s e ~ C S 50149, ~} 60201$ Compiègne Cedex, France
}

Supporting Information Placeholder

\begin{abstract}
The in situ preparation of highly stereoretentive ruthenium-based metathesis catalysts is reported. This approach completely avoids the isolation of intermediates and air-sensitive catalysts, thus allowing for the rapid access and evaluation of numerous dithiolate Ru-catalysts. A procedure was established to perform cross-metathesis reactions without the use of a glove box, and on small scale even Schlenk techniques are not required. Consequently, the chemistry displayed in this report is available to every practicing organic chemist and presents a powerful approach for the identification of new stereoretentive catalysts.
\end{abstract}

Catalytic methods for the synthesis of olefins with high stereochemical purity are in high demand, particularly if these transformations produce less waste vis-à-vis established stoichiometric procedures. ${ }^{1,2}$ Recently, a number of efficient Z-selective Ru-based metathesis catalysts have been reported by Grubbs and Jensen., ${ }^{3,4}$ These catalysts transform terminal alkenes to Z-alkenes with high stereoselectivity. In 2013, Hoveyda er al. reported the first highly stereoretentive ruthenium dithiolate catalysts $\mathbf{R u - 1}$ and $\mathbf{R u}-2 .{ }^{5}$ Two years later, the more robust dichlorosubstituted catalyst $\mathrm{S}_{2} \mathbf{R u}-\mathbf{3}$ was identified by the same group. ${ }^{6}$ These catalysts allow for the transformation of $E$ - or Z-olefins with high retention of the original stereochemical information (Scheme $1 \mathrm{~b}$, eq 1$)^{2 \mathrm{~d}}$ The great functional group tolerance of ruthenium dithiolate catalysts combined with the high stereochemical purities obtained for the products has given rise to a remarkable research activity in this area over the past five years. ${ }^{7}$ Independent investigations by Hoveyda and Grubbs highlight the importance of electronic and steric properties of the NHC- and dithiolate ligand for the efficiency of ruthenium dithiolate catalysts. ${ }^{6,7 \mathrm{e}-\mathrm{f}, 8}$ The origins of stereoretentive mechanism have been recently elucidated by computational studies by Liu and Houk. ${ }^{9}$ Given the many advantageous of stereoretentive dithiolate ruthenium catalysts, important progress would be made if this class of catalyst could be made available to every organic chemist in any desired quantity.

Scheme 1. Previous work by Hoveyda et al. and this work

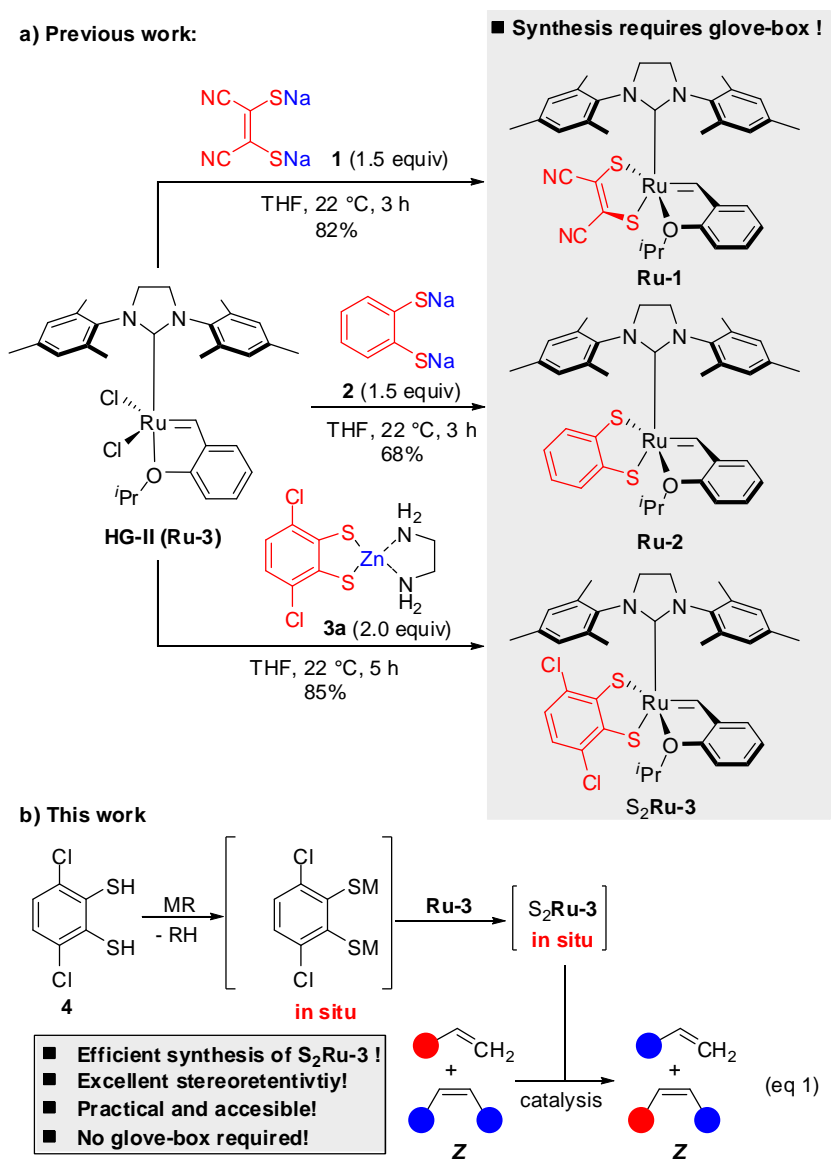

Currently $\mathbf{R u}-\mathbf{1}, \mathbf{R u}-\mathbf{2}$ and $\mathbf{S}_{\mathbf{2}} \mathbf{R u}-\mathbf{3}$ are synthesized via a two step procedure, requiring manipulation in the glove-box (Scheme 1a). ${ }^{5,6}$ First, the corresponding disodium $(\mathbf{1} \text { and } 2)^{5}$ or zincdithiolate (3) ${ }^{6}$ salt is prepared from the commercially available dithiols by deprotonation with either $\mathrm{NaOtBu}$ or $\mathrm{Zn}(\mathrm{OAc})_{2}$ in the presence of ethylenediamine. Second, commercially available Hoveyda-Grubbs catalyst Ru-3 is reacted with $\mathbf{1}, \mathbf{2}$ or $\mathbf{3 a}$ to give, after filtration in a glove box, the corresponding air sensitive dithiolate catalysts. ${ }^{6,10}$

Stimulated by our recent involvement in the field of stereoselective and stereoretentive metathesis catalysts, ${ }^{7 c, 11}$ we became 
interested in the development of a highly practical and easily accessible stereoretentive metathesis catalyst. Due to our strong background in copper catalyzed reactions, ${ }^{12}$ where the catalyst is often generated in situ by mixing the corresponding ligand and the $\mathrm{Cu}$-salt, we wondered if a related process would be possible for the in situ generation of dithiolate catalysts (Scheme 1b). We surmised that the right choice of a base to deprotonate dithiol 4 might allow for the efficient in situ generation of catalyst $\mathbf{S}_{2} \mathbf{R u}-\mathbf{3}$ while generating only side products which do not perturb the catalytic activity of the metathesis catalyst. Furthermore, we speculated that solutions of the in situ generated catalyst might be stable enough to tolerate semiinert conditions (argon-flushed vials), hence allowing for the reaction to be carried out outside of a glove-box without the use of Schlenk-equipment. If such a simple and practical procedure could be implemented, the benefits of ruthenium dithiolate catalysts would be available to the entire organic synthetic community.

With these goals in mind, we started out by evaluating several bases for the in situ generation of metal dithiolates (Scheme 2). Subsequently, we added Ru-3 to the metal dithiolate solutions to generate in situ (IS) $\mathbf{S}_{\mathbf{2}} \mathbf{R u} \mathbf{- 3}$. The catalyst solution was immediately subjected to the cross-metathesis (CM) of cisbutenediol 5 and 1-dodecene $\mathbf{6}$.

Scheme 2. Scouting studies; identification of $\mathrm{Et}_{2} \mathrm{Zn}$ for efficient synthesis of $\mathrm{S}_{2} \mathrm{Ru}-3$.

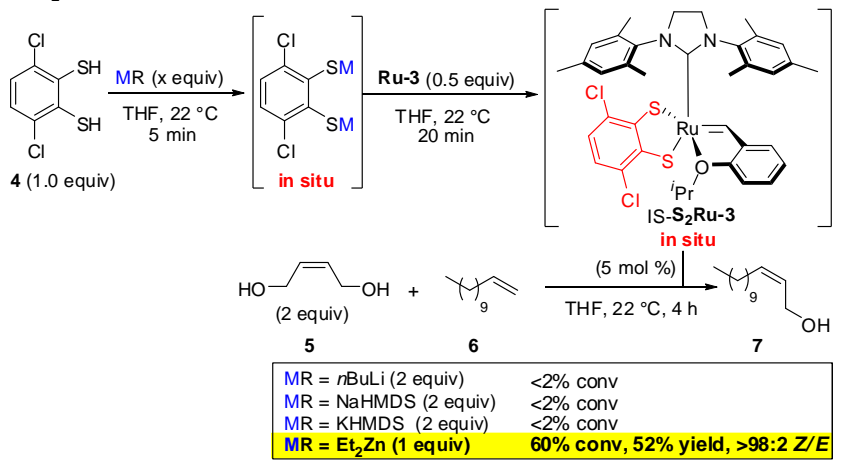

The outcome of our scouting studies clearly showed the challenge of our in situ approach. $n$-BuLi, NaHMDS and KHMDS gave completely inefficient $\mathrm{CM}$, probably due to incomplete formation of $I S-\mathbf{S}_{\mathbf{2}} \mathbf{R u}-\mathbf{3}$ or generation of deleterious byproducts. ${ }^{13}$ In stark contrast, $\mathrm{Et}_{2} \mathrm{Zn}$ allowed for synthesis of allylic alcohol $\mathbf{7}$ with excellent stereoretention (>98:2 Z-selectivity). ${ }^{14}$ Importantly, excess of zinc-dithiolate 8 and generated $\mathrm{ZnCl}_{2}$ had only a modest affect on the reaction outcome compared to the isolated catalyst (Table 1, entries 12 ). Carrying out the same reaction in the glove box gave slightly increased yields with identical stereochemical purity (entry 3). We took advantage of the simple in situ synthesis of ruthenium dithiolate catalysts to survey the efficiency of numerous, mostly commercially available catalysts for the CM of 5 and 6 (Fig. 1 and Table 1).

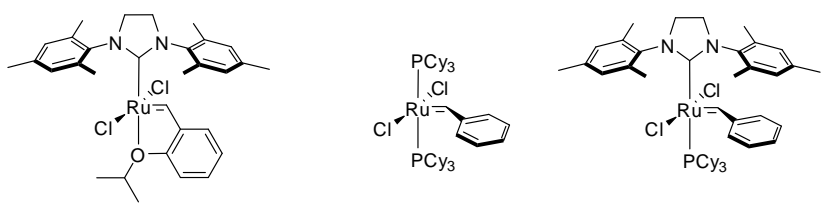

Ru-3 (Hoveyda-Grubbs II)

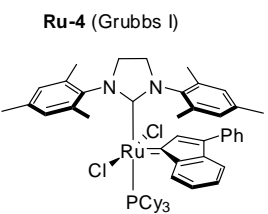

Ru-5 (Grubbs II)

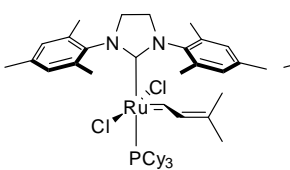

Ru-6 (Grubbs vinylidene)

Ru-7 (Umicore M2)
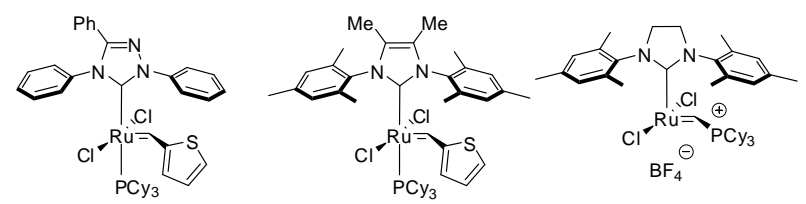

Ru-9 (Evonik RF4)

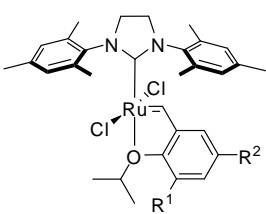

Ru-10 (Evonik RF3)

Ru-11 (Piers)

$\mathrm{Ru}-12, \mathrm{R}^{1}=\mathrm{H}, \mathrm{R}^{2}=\mathrm{NO}_{2}$

(Grela)

Ru-13, $\mathrm{R}^{1}=\mathrm{Ph}, \mathrm{R}^{2}=\mathrm{H}$

(Blechert)

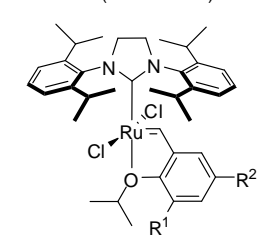

Ru-14, $\mathrm{R}^{1}=\mathrm{H}, \mathrm{R}^{2}=\mathrm{NO}_{2}$ Ru-15, $R^{1}=P h, R^{2}=H$ Ru-16, $R^{1}=R^{2}=H$

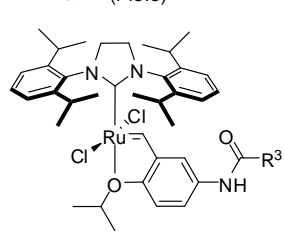

Ru-17, $\mathrm{R}^{3}=\mathrm{CF}_{3}$ (Umicore M71) $\mathrm{Ru}-\mathbf{1 8}, \mathrm{R}^{3}=\mathrm{O} i-\mathrm{Bu}$ (Umicore M73)
Figure 1. Ru-based metathesis catalysts used in this study.

Phosphine based catalysts afforded 7 either in poor yield or with poor stereochemical purity (Table 1, entries 4-10). Exposing 14-electron Piers complex (Ru-11) to our in situ procedure, and cross-metathesis reaction furnished 7 with excellent stereoselectivity (Table 1, entry 11). Importantly, no study concerning 14-electron ruthenium dithiolate catalysts was reported in the literature. ${ }^{8 a}$ Attempts to isolate the corresponding dithiolate catalyst however failed, very likely due to the lack of stability of the corresponding complex. Nevertheless, we were encouraged by this result, as the in situ strategy allowed for the identification of a previously unknown catalyst. Precursors of the Hoveyda-type catalysts (Ru-16), including the well known Grela (Ru-12 and Ru-14) and Blechert (Ru13 and Ru-15) derivatives as well as our in house developed catalysts (Ru-17 and Ru-18) ${ }^{15}$ afford the CM-product $\mathbf{7}$ in modest to good yields with constantly high stereochemical puritiy (Table 1, entries 8-15). Further optimization showed that a minimum of 1.5 equivalents of dithiolate zinc reagent 2 has to be added to the catalyst precursor in order to afford high levels of stereoselectivity (Table 1, entries 19-21). We were aware of the fact that terminal alkenes such as alkene $\mathbf{6}$ resulted in catalyst decomposition. ${ }^{6}$ Indeed, portion wise addition of catalyst $I S-\mathbf{S}_{\mathbf{2}} \mathbf{R u}-\mathbf{3}$ resulted in an approximate $10 \%$ increase in product yield (Table 1, entries 22-23). With the optimized conditions (Table 1, entry 23) in hand we started exploring the scope of the reaction (Scheme 3). 
Table 1: Screening of ruthenium dichloride precursors for the in situ generation of Ru-dithiolate catalysts ${ }^{a}$

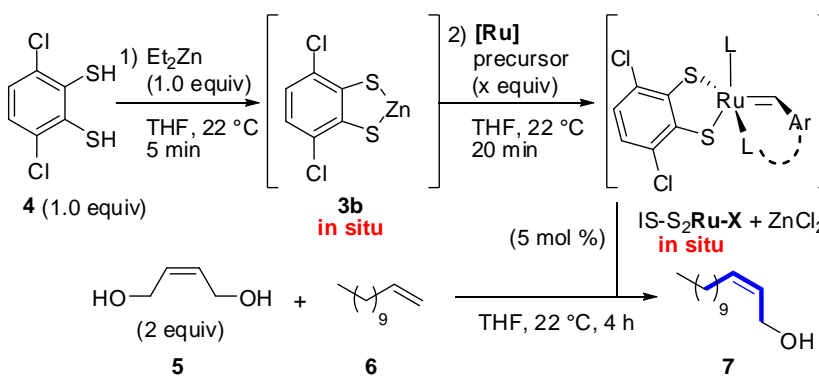

\begin{tabular}{|c|c|c|c|c|c|}
\hline entry & $\begin{array}{c}\text { [Ru] precursor } \\
(\mathrm{x} \text { equiv.) }\end{array}$ & $\begin{array}{c}\text { catalyst } \\
(5 \mathrm{~mol} \%)\end{array}$ & conv. $[\%]^{\mathrm{b}}$ & yield $[\%]^{c}$ & $Z / E^{\mathrm{d}}$ \\
\hline $1^{\mathrm{e}}$ & -- & $\mathrm{S}_{2} \mathrm{Ru}-\mathbf{3}$ & 81 & 72 & $96: 4$ \\
\hline 2 & Ru-3 (0.5) & IS-S ${ }_{2} \mathrm{Ru}-3$ & 60 & 52 & $>98: 2$ \\
\hline $3^{f}$ & Ru-3 (0.5) & $I S-S_{2} R u-3$ & 70 & 61 & $>98: 2$ \\
\hline 4 & $\mathrm{Ru}-4(0.5)$ & $I S-S_{2} R u-4$ & 13 & n.d. & n.d. \\
\hline 5 & Ru -5 (0.5) & $I S-S_{2} R u-5$ & 55 & 48 & $56: 44$ \\
\hline 6 & Ru-6 (0.5) & $I S-S_{2} R u-6$ & 15 & n.d. & n.d. \\
\hline 7 & Ru-7 (0.5) & $I S-S_{2} R u-7$ & 57 & 51 & $20: 80$ \\
\hline 8 & Ru-8 (0.5) & $I S-S_{2} R u-8$ & 35 & 25 & $26: 74$ \\
\hline 9 & Ru -9 (0.5) & IS-S ${ }_{2} \mathrm{Ru}-9$ & 45 & 35 & $17: 83$ \\
\hline 10 & Ru-10 (0.5) & $I S-S_{2} R u-10$ & 16 & n.d. & n.d \\
\hline 11 & Ru-11 (0.5) & IS-S ${ }_{2}^{R u-11}$ & 37 & 35 & $>98: 2$ \\
\hline 12 & Ru-12 (0.5) & $I S-S_{2} R u-12$ & 52 & 37 & $>98: 2$ \\
\hline 13 & Ru-13 (0.5) & $I S-S_{2} R u-13$ & 38 & 26 & $>98: 2$ \\
\hline 14 & Ru-14 (0.5) & IS-S ${ }_{2} \mathrm{Ru}-14$ & 59 & 41 & $>98: 2$ \\
\hline 15 & Ru-15 (0.5) & IS-S ${ }_{2} \mathrm{Ru}-15$ & 48 & 34 & $94: 6$ \\
\hline 16 & Ru-16 (0.5) & $I S-S_{2} R u-16$ & 63 & 49 & $96: 4$ \\
\hline 17 & Ru-17 (0.5) & IS-S ${ }_{2} \mathrm{Ru}-17$ & 55 & 48 & $98: 2$ \\
\hline 18 & Ru-18 (0.5) & $I S-S_{2} R u-18$ & 73 & 61 & $95: 5$ \\
\hline 19 & Ru-3 (0.66) & $I S-S_{2}$ Ru -3 & 62 & 53 & $98: 2$ \\
\hline 20 & Ru-3 (0.85) & $I S-S_{2} R u-3$ & 66 & 55 & $86: 14$ \\
\hline 21 & Ru-3 (1) & $I S-S_{2} R u-3$ & 58 & 45 & $62: 38$ \\
\hline $22^{g}$ & Ru-3 (0.66) & IS-S ${ }_{2} \mathrm{Ru}-3$ & 62 & 55 & $>98: 2$ \\
\hline $23^{g, h}$ & Ru-3 (0.66) & $I S-S_{2} R u-3$ & 74 & 64 & $>98: 2$ \\
\hline
\end{tabular}

${ }^{a}$ Reactions performed in argon flushed vials on a $0.4 \mathrm{mmol}$ scale. ${ }^{b}$ Determined by GC. ${ }^{c}$ Isolated yield. ${ }^{d}$ Determined by ${ }^{1} \mathrm{H}-\mathrm{NMR}$. ${ }^{e}$ Result obtained by Hoveyda and co-workers (see ref 6). ${ }^{f}$ Reaction carried out in a glove-box with degassed solvents. n.d. = not determined. ${ }^{g}$ With 6 mol \% of $I S-S_{2} \mathbf{R u}-3$. ${ }^{h}$ Addition of catalyst in four equal portions (1.5 mol \% after each hour).

The great variety of products which were obtained attests to the robustness of the in situ generated catalyst $I S-\mathbf{S}_{2} \mathbf{R u}-\mathbf{3}$. Even in the presence of $3 \%$ of $\mathrm{Zn}$-dithiolate and $6 \%$ of $\mathrm{ZnCl}_{2}$ which are likely to react with some of the functional groups, the in situ generated catalyst still afforded useful synthetic yields which are in most instances similar to the ones obtained with the isolated catalyst $\mathbf{S}_{\mathbf{2}} \mathbf{R u} \mathbf{- 3}$ and in some cases even higher (e.g. 14 and 20). ${ }^{6}$ Noteworthy is the tolerance toward sensitive functional groups such as bromides $\mathbf{1 0}$ and aldehydes $\mathbf{1 1}$. Bromide 10 was also prepared on larger scale $(4.5 \mathrm{mmol})$ with continuous addition of only two mole percent of catalyst over two hours with a syringe-pump. We were pleased to observe a significantly higher turn over number of 21 compared to the portion wise addition (TON of 12). Acidic functional groups such as carboxylic acid $\mathbf{1 2}$ or phenol $\mathbf{1 5}$ perform particularly well. Diester 16 was synthesized in 53\% yield, comparing favorably to a recent four-step synthesis thereof (23\% overall yield). ${ }^{16}$ Sterically hindered alkenes (leading to products $\mathbf{1 7}$ and 18) reacted with excellent levels of selectivity, albeit in slightly lower yields. The cross-metathesis of styrenes was strongly dependent on its electronic properties. While the reaction with styrene was poor yielding (19), electron poor 4-(trifluoromethyl)styrene afforded 20 in good yield and high levels of $Z$-selectivity. The cross-metathesis with cis-diacetoxy-2-butene yielded $\mathbf{2 1}$ in good yields and high selectivity. ${ }^{\text {7h, } 17}$ In terms of limitations we noticed a significant drop in yield for allyl ether $\mathbf{2 2}$ and disubstituted alkenes such as $(R)$ limonene 23.

Scheme 3. Scope of the stereoretentive metathesis reaction. (See SI for details).

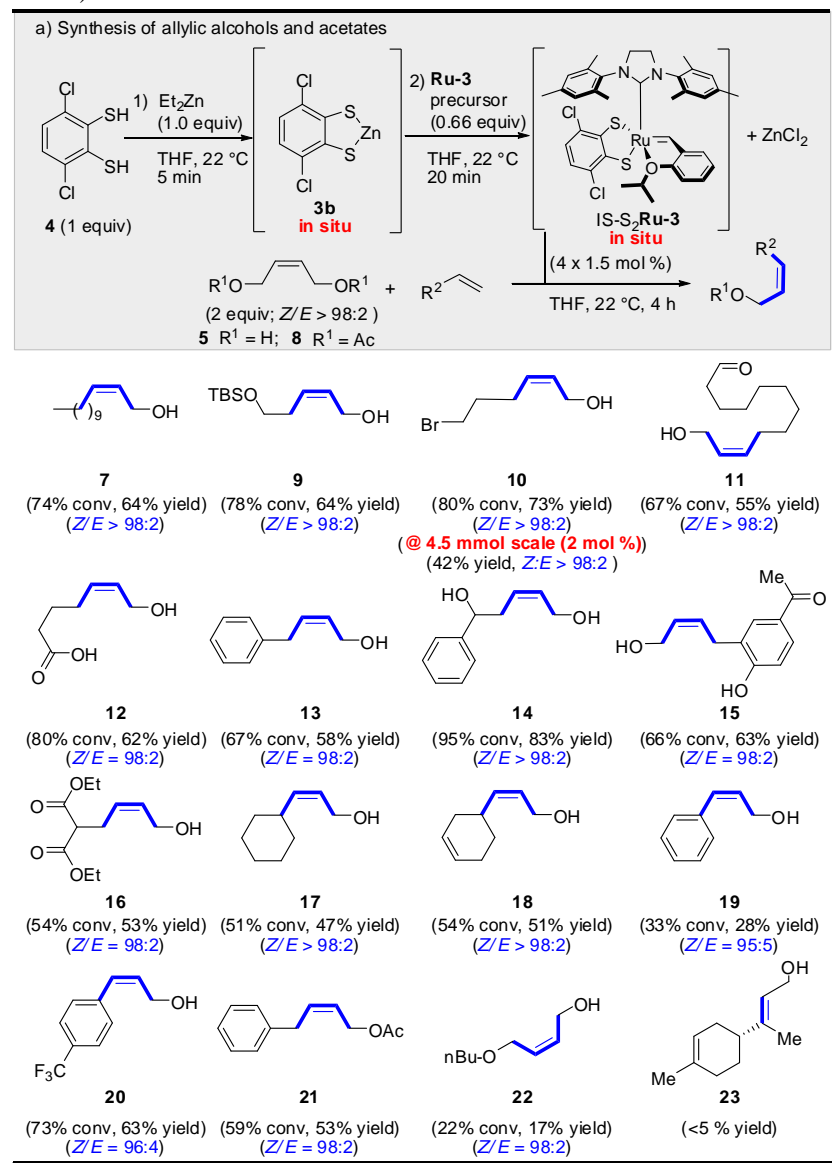

b) Highly $Z$-stereoretentive synthesis of 16 -membered macrocycle 25

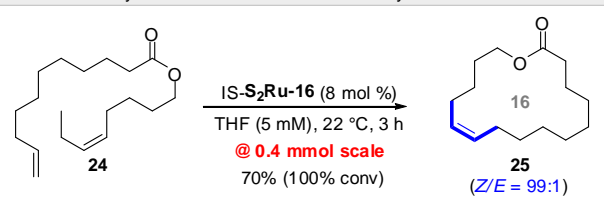

c) Stereoretentive self-metathesis of $(Z)$ and $(E)$-methyl-9-decenoate 26

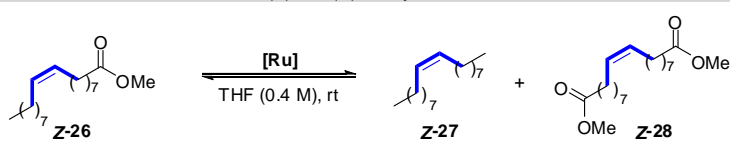

Z-26 (99\%); IS-Ru-15 (0.01 mol \%), $16 \mathrm{~h}$ to equilibrium (Z:E > 99:1)

Z-26 (85\%); IS-Ru-16 (0.1 mol \%), $2 \mathrm{~h}$ to equilibrium ( $Z: E=98: 2)$; $@ 8.0 \mathrm{mmol}$ scale)

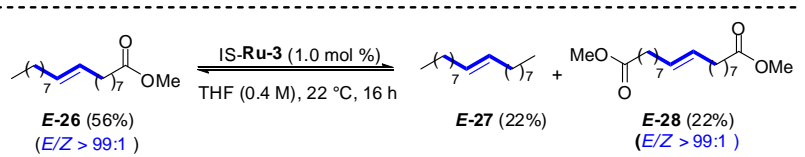

Next, we tested in situ generated catalysts for stereoretentive ring-closing metathesis (Scheme 3b). After a preliminary screening of catalysts (See Supporting Information (SI)) we found in situ generated $I S-\mathbf{S}_{\mathbf{2}} \mathbf{R u}-\mathbf{1 6}$ to give macrocyle $\mathbf{2 5}$ with excellent selectivity and $70 \%$ yield. We also tested a selection of in situ generated Ru-dithiolate catalysts for the selfmetathesis of Z- and E-methyl 9-octadecenoate (26) to produce 9-octadecene (27) and dimethyl 9-octadecenedioate (28) (Scheme 3c and SI). For the reaction with Z-26 (99\% purity) the equilibrium (26:27:28 $=50: 25: 25)$ was achieved with only 
$100 \mathrm{ppm}$ of $I S-\mathbf{S}_{\mathbf{2}} \mathbf{R u}-\mathbf{1 5}$ with excellent stereochemical purity ( $Z: E>99: 1)$. Carrying out the self-metathesis reaction with technical grade methyl oleate $85 \%$ purity (Radia $7072^{\circledR}$ ) demonstrated the robustness of the in situ protocol described herein. ${ }^{18}$ Within only two hours the equilibrium was achieved with high stereochemical purity of the components $(Z / E=$ 98:2). The self-metathesis of $E$-methyl 9-octadecenoate (E-26) proceeded smoothly with excellent stereoretention in the presence of catalyst $I S-\mathbf{S}_{2} \mathbf{R u}-\mathbf{3}$ (Scheme 3c).

In conclusion, we have developed a highly practical in situ protocol for the generation of stereoretentive Ru-based metathesis catalysts. These catalysts perform very well, often delivering the products in similar yields and selectivity compared to the isolated catalysts. Furthermore, the in situ protocol allowed for the rapid evaluation of a great number of dichloro-ruthenium precursors. This enabled the identification of a previously unknown ditholate-ruthenium catalyst derived from the Piers-catalyst Ru-11. Our findings concerning the evaluation of new ligands leading to stereoretentive Rucatalysts using the in situ method described herein will be reported in due course.

\section{ASSOCIATED CONTENT}

\section{Supporting Information}

Optimization reactions, experimental procedures and characterization data $\left({ }^{1} \mathrm{H}\right.$ NMR, ${ }^{13} \mathrm{C}$ NMR, GC-traces). This material is available free of charge via the Internet at http://pubs.acs.org.

\section{AUTHOR INFORMATION}

Corresponding Author

marc.mauduit@ensc-rennes.fr

The authors declare no competing financial interest.

\section{ACKNOWLEDGMENTS}

This work was supported by the FASO (grant to DSM and IC; ZSELECT) and the Région Bretagne (SAD $2016 \mathrm{~N}^{\circ} 9639-$ RZSELECT; grant to OB and DSM). OB and MM acknowledge the Ecole Nationale Supérieure de Chimie de Rennes (ENSCR) and the Centre National de la Recherche Scientifique (CNRS) for financial support.

\section{REFERENCES}

(1) For a recent review concerning the stereoselective synthesis of Z-alkenes via traditional methods, see: W. Siau, Y. Zhang, Y. Zhao, Top. Curr. Chem. 2012, 327, 33-58.

(2) For excellent reviews on stereoselective and stereoretentive metathesis, see: a) A. H. Hoveyda, J. Org. Chem. 2014, 79, 4763-4792; b) S. Werrel, J. C. L. Walker, T. J. Donohoe, Tet. Lett. 2015, 56, 5261-5268; c) M. H. Herbert, R. H. Grubbs, Angew. Chem. Int. Ed. 2015, 54, 5018-5024; d) T. P. Montgomery, T. S. Ahmed, R. H. Grubbs, Angew. Chem. Int. Ed. 2017, 56, 11024 - 11036; e) T. P. Montgomery, A. M. Johns, R. H. Grubbs, Catalysts 2017, 7, 87-124.

(3) L. E. Rosenbrugh, M. B. Herbert, V. M. Marx, B. K. Keitz, R. H. Grubbs, J. Am. Chem. Soc. 2013, 135, 1276-1279.

(4) G. Occhipinti, F. R. Hansen, K. W. Törnroos, V. R. Jensen, J. Am. Chem. Soc. 2013, 135, 3331-3334.

(5) R. K. M. Khan, S. Torker, A. H. Hoveyda, J. Am. Chem. Soc. 2013, 135, 10258-10261. Sodium maleonitriledithiolate for the synthesis of Ru-1 is commercially available (CAS: 5466-54-6).

(6) M. J. Koh, R. K. M. Khan, S. Torker, M. Yu, M. S. Mikus, A. H. Hoveyda, Nature 2015, 517, 181-186.

(7) For applications in ROMP, see ref. 5 and a) M. S. Mikus, S. Torker, A. H. Hoveyda, Angew. Chem. Int. Ed. 2016, 55, 4997-5002.
For applications in ring-opening/cross-metathesis (ROCM), see ref. 5, b) M. J. Koh, R. K. M. Khan, S. Torker, A. H. Hoveyda, Angew. Chem. Int. Ed. 2014, 53, 1968 -1972 and c) A. Dumas, D. S. Müller, I. Curbet, L. Toupet, M. Rouen, O. Baslé, M. Mauduit, Organometallics 2018, 37, 829-834. For applications in cross and self-metathesis, see: ref. 6, 7c, d) M. Yu, R. R. Schrock, A. H. Hoveyda, Angew. Chem. Int. Ed. 2015, 54, 215 -220, e) A. M. Johns, T. S. Ahmed, B. W. Jackson, R. H. Grubbs, R. L. Pederson, Org. Lett. 2016, 18, $772-775$, f) T. S. Ahmed, R. H. Grubbs, J. Am. Chem. Soc. 2017, 139, 1532-1537, g) Xu, S. Shen, A. H. Hoveyda, J. Am. Chem. Soc. 2017, 139, 10919-10928 and h) C. Xu, Z. Liu, S. Torker, X. Shen, D. Xu, A. H. Hoveyda, J. Am. Chem. Soc. 2017, 139, 15640-15643. For applications in ring-closing metathesis (RCM), see: 7c, 7g, i) T. S. Ahmed, R. H. Grubbs, Angew. Chem. Int. Ed. 2017, 56, 11213 11216 and j) T. S. Ahmed, T. P. Montgomery, R. H. Grubbs, Chem. Sci. 2018, 3580-3583.

(8) a) M. S. Mikus, S. Torker, C. Xu, B. Li, A. H. Hoveyda, Organometallics 2016, 35, 3878-3892. b) T. P. Montgomery, J. M Grandner, K. N. Houk, R. H. Grubbs, Organometallics 2017, 36, 3940-3953.

(9) J. M. Grandner, H. Shao, R. H. Grubbs, P. Liu, K. N. Houk, J. Org. Chem. 2017, 82, 10595-10600.

(10) Hoveyda reported that reactions with $\mathbf{S}_{2} \mathbf{R u}-\mathbf{3}$ can be stopped by addition of technical grade ether (wet ether), see ref. 6 . Our results concerning the RCM of $\mathbf{2 4}$ showed that the catalyst degrades in the presence of air. Reactions carried out with non-degassed THF gave in the presence of $10 \mathrm{~mol} \%$ of catalyst conversions $<2 \%$.

(11) A. Dumas, R. Tarrieu, T. Vives, T. Roisnel, V. Dorcet, O. Baslé, M. Mauduit, ACS Catal. 2018, 8, 3257-3262.

(12) For recent contributions of our group in the field of $\mathrm{Cu}-$ catalyzed reactions, see: a) R. Tarrieu, A. Dumas, J. Thongpaen, T. Vives, T. Roisnel, V. Dorcet, C. Crévisy, O. Baslé, M. Mauduit, J. Org. Chem. 2017, 82, 1880-1887; b) S. Drissi-Amraoui, T. E. Schmid, J. Lauberteaux, C. Crévisy, O. Baslé, R. Marcia de Figueiredo, S. Halbert, H. Gérard, M. Mauduit, J.-M. Campagne, Adv. Synth. Catal. 2016, 358, 2519-2540; c) S. Drissi-Amraoui, M. S. T. Morin, C. Crévisy, O. Baslé, R. Marcia de Figueiredo, M. Mauduit, J.-M. Campagne, Angew. Chem. Int. Ed. 2015, 54, 11830-11834.

(13) Hoveyda reported poor yields of $\mathbf{S}_{2} \mathbf{R u} \mathbf{3} \mathbf{3}$ when the disodium dithiolate salt was used for its synthesis (ref. 6).

(14) The complete deprotonation of dithiol 4 by $\mathrm{Et}_{2} \mathrm{Zn}$ was confirmed by ${ }^{1} \mathrm{H}-\mathrm{NMR}$ analysis (See SI).

(15) For a recent account covering the contributions of this group in the field of Ru-catalyzed metathesis reactions: T. E. Schmid, A. Dumas, S. Colombel-Rouen, C. Crévisy, O. Baslé, M. Mauduit, Synlett 2017, 28, 773-798.

(16) M. Chiarucci, M. Locritani, G. Cera, M. Bandini, Beilstein J. Org. Chem. 2011, 7, 1198-1204

(17) Hence a hydrogen-bond is not required as recently discussed by Hoveyda (ref. 7h). For an excellent review concerning the allylic chalcogen effect in metathesis, see: M. Chiarucci, M. Locritani, G. Cera, M. Bandini, Beilstein J. Org. Chem. 2011, 7, 1198-1204.

(18) We recently reported on the self-metathesis of Radia ${ }^{\circledR}$ on large scale: J. Allard, I. Curbet, G. Chollet, F. Tripoteau, S. Sambou, F. Caijo, Y. Raoul, C. Crévisy, O. Baslé, M. Mauduit, Chem. Eur. J. 2017, 23, $12729-12734$. 
Insert Table of Contents artwork here

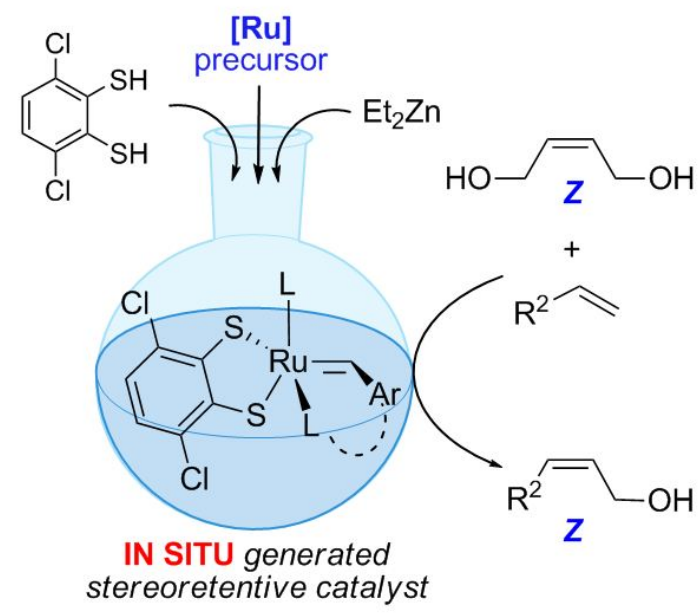

Commercially available starting materials

Bench procedure (without glove-box)

16 precatalyst screened

Highly Z- or $E$ -

stereoretentive (up to >98:2)

- 19 examples

Catalyst loading as low as 100 ppm 